\title{
Predictors of Mild Cognitive Impairment in Early-Stage Parkinson's Disease
}

\author{
Brenda Hanna-Pladdy ${ }^{a}$ b Katherine Jones ${ }^{c}$ Romeo Cabanban ${ }^{a}$ \\ Rajesh Pahwad Kelly E. Lyons ${ }^{d}$ \\ a Division of Neuropsychology, Department of Neurology, and ${ }^{\mathrm{b}}$ Department of Radiology \\ and Imaging Sciences, Emory University School of Medicine, Atlanta, Ga., and \\ 'Landon Center on Aging, and dParkinson's Disease and Movement Disorder Center and \\ Department of Neurology, University of Kansas Medical Center, Kansas City, Kans., USA
}

\author{
Key Words \\ Parkinson's disease $\cdot$ Mild cognitive impairment $\cdot$ Predictors $\cdot$ Neuropsychological \\ performance $\cdot$ Dopaminergic medication
}

\begin{abstract}
Aim: The aim of this study was to identify mild cognitive deficits in Parkinson's disease (PD) prior to extensive neurodegeneration and to evaluate the extent to which dopamine depletion and other disease-related predictors can explain cognitive profiles. Methods: Neuropsychological performances of 40 nondemented early-stage PD patients and 42 healthy controls were compared across on or off dopaminergic medications. Stepwise regression evaluated cognitive predictors of early-stage PD and disease-related predictors of PD cognition (levodopa dose, disease duration, Unified Parkinson's Disease Rating Scale score, sleep, quality of life, and mood) across on and off states. Results: Neuropsychological performance was lower in PD patients across cognitive domains with significant memory, naming, visuomotor, and complex attention/executive deficits, but with intact visuospatial, simple attention, and phonemic fluency functions. However, medication effects were absent except for simple attention. Regression analyses revealed age, working memory, and memory recall to be the best cognitive predictors of PD, while age, quality of life, disease duration, and anxiety predicted PD cognition in the off state. Conclusion: Nondemented early-stage PD patients presented with extensive mild cognitive deficits including prominent memory impairment. The profile was inconsistent with expected isolated frontostriatal dysfunction previously attributed to dopamine depletion and this highlights the need to further characterize extranigral sources of mild cognitive impairment in PD.

Copyright (C) 2013 S. Karger AG, Basel
\end{abstract}


Parkinson's Disease

\section{Introduction}

Parkinson's disease (PD) has traditionally been defined by cardinal motor features of tremor, rigidity, bradykinesia, and postural instability in the later stages, and has been attributed to dopamine deficiency reflective of degeneration in the nigrostriatal system $[1,2]$. There is increased emphasis on identifying premotor symptoms of PD when nonmotor features such as mild cognitive changes might characterize the earliest phases of the disease, prior to the stage of extensive neurodegeneration resulting in motor impairment $[3,4]$. Although cognitive impairment in PD has been well accepted and a high incidence of dementia in the later stages has been demonstrated, the characteristic profiles suggestive of the underlying pathophysiological mechanisms remain unclear $[5,6]$. Therefore, the extent to which dopamine depletion can explain mild cognitive deficits during the early stage of PD is of critical importance to elucidate neural mechanisms mediating the preclinical phase of PD and the pathophysiology of nonmotor features of the disease.

Although neuropsychological deficits in PD have been clearly documented, the characteristic profiles based on disease duration and the underlying neuropathology implicated remain controversial $[5,7,8]$. This is especially the case when reviewing the literature on dopamine replacement therapy in cognition. Study findings have been inconsistent across cognitive task demands and may be partly related to failure to control for disease severity and daily levodopa doses [9-11]. Nonetheless, mild cognitive impairment in PD is well accepted, and early cognitive impairment has demonstrated predictive validity for a later conversion to dementia as well as reductions in quality of life $[7,12,13]$. Clinically, the expected cognitive profile of patients with PD has been described as a 'subcortical syndrome' with greater impairment in executive and attentional functions and less impairment in memory, language, and visuospatial functions, presumably related to the involvement of the frontostriatal system [14]. However, cognitive deficits in PD are heterogeneous, with some patients displaying memory deficits that may place them at greater risk for the development of dementia in the later stages of the disease, raising the question of whether extranigral pathology is implicated in early cognitive decline [12,15-17]. Therefore, clear characterization of neuropsychological profiles early in the disease process is critical to elucidate the neuropathological basis of PD. Moreover, this will facilitate the identification of new therapeutic targets focused on treating the entire constellation of motor and nonmotor PD symptoms that are more likely to affect both the onset and progression of symptoms, resulting in disease-related disability [18].

Evidence supporting the hypothesis that structural changes are present in the earliest stages of the disease is emerging, but the relationship between neuroimaging changes and neuropsychological deficits has not been studied extensively. While neocortical atrophy has been described in PD patients with dementia, there may also be structural changes in cortical and subcortical regions in PD patients without dementia underlying mild cognitive deficits traditionally attributed primarily to frontostriatal dysfunction assumed to result from dopamine deficiency $[19,20]$. Cortical thinning has been identified as being particularly pronounced in frontotemporal regions in early stages of PD, while regional cerebral glucose metabolism has implicated extensive hypometabolism in temporoparietal regions in PD patients with mild cognitive impairment [21,22]. Consequently, there is growing evidence of the presence of extranigral pathology that likely occurs well before dopamine depletion, although it remains unclear which cognitive deficits can be attributed to dopaminergic loss as opposed to structural degeneration [18-20].

To further elucidate the role of dopamine in cognitive deficits in PD, we evaluated the neuropsychological profile of nondemented early-stage PD patients compared to that of normal healthy controls and investigated the degree of dopaminergic modulation by evalu- 
Hanna-Pladdy et al.: Predictors of Mild Cognitive Impairment in Early-Stage Parkinson's Disease

Table 1. Mean demographics and screening measures (SD) for the PD and control groups

\begin{tabular}{lllllll}
\hline Group & $\begin{array}{l}\text { Age } \\
\text { years }\end{array}$ & $\begin{array}{l}\text { Education } \\
\text { years }\end{array}$ & MMSE score & EDIN score & BAI score & BDI-II score \\
\hline Control $(\mathrm{n}=42)$ & $66.9(4.6)$ & $16.3(1.5)$ & $29.3(0.7)$ & $86.9(11.0)$ & $1.2(1.4)$ & $3.3(2.5)$ \\
PD $(\mathrm{n}=40)$ & $66.0(7.7)$ & $15.3(2.6)$ & $28.6(1.3)$ & $75.9(45.7)$ & $9.6(8.3)$ & $9.5(6.8)$ \\
\hline
\end{tabular}

Table 2. Mean scores (SD) for PD patients in the on and off medication states

\begin{tabular}{llllllll}
\hline Variable & UPDRS total & UPDRS motor & EP & PDSS & BDI-II & BAI & PDQ-39 \\
\hline On & $30.5(11.1)$ & $18.7(6.2)$ & $11.4(5.2)$ & $104.2(21.7)$ & $9.3(6.3)$ & $9.8(9.5)$ & $20.8(15.5)$ \\
Off & $43.5(13.3)$ & $29.4(8.8)$ & $10.7(5.2)$ & $101.7(23.9)$ & $9.7(7.7)$ & $9.4(15.5)$ & $22.2(15.6)$ \\
\hline
\end{tabular}

ating patients both on and off dopaminergic medications. In addition to investigating the role of dopamine in cognition, we also evaluated other disease-related predictors (quality of life, levodopa daily dosage, motor impairment, age, etc.) of mild cognitive deficits in early-stage PD.

\section{Methods}

\section{Subjects}

The study population ( $\mathrm{n}=82)$ consisted of 40 nondemented early-stage PD patients and 42 age- and education-matched normal controls. Healthy controls were recruited from the Landon Center on Aging database and patients from the Parkinson's Disease and Movement Disorder Center at the Kansas University Medical Center. General enrollment criteria for healthy controls were as follows: (i) age 50-75 years; (ii) right-handed defined as a score of $>60$ on the Edinburgh Handedness Inventory (EDIN) [23]; (iii) a minimum score of 26 out of 30 on the Mini-Mental State Examination (MMSE) [24], and (iv) no dementia as determined by the Repeatable Battery for the Assessment of Neuropsychological Status (RBANS; table 1) [25]. General exclusion criteria included a history of neurologic disorder other than PD, dementia, major psychiatric disorder (including alcohol or substance abuse), concurrent, unstable, or serious medical condition, major head trauma, chronic use of psychoactive medications, and the presence of dyskinesia based on neurological examination. The study was conducted in accordance with the Declaration of Helsinki and was approved by the Institutional Review Boards of the Kansas University Medical Center and the Emory University School of Medicine. All participants gave their written informed consent.

Selection criteria for PD subjects included a diagnosis of idiopathic PD based on the United Kingdom Parkinson's Disease Society Brain Bank Criteria as well as on the criteria proposed by Hughes et al. [26]. Subjects selected had mild disease severity based on a Hoehn and Yahr rating of $\leq 2.5$, a Unified Parkinson's Disease Rating Scale (UPDRS) motor score of $\leq 20$ or a UPDRS total score of $\leq 30$ in the on medication state (see table 2 for on and off medication values), and a mean disease duration of 5.7 years ( $\mathrm{SD}=2.7$ years). All patients were prescribed levodopa with an average daily dosage of $597.5 \mathrm{mg}(\mathrm{SD}=288.7)$ as well as one of two dopamine agonists (pramipexole $=47.5 \%$; ropinirole $=52.5 \%$ of the PD group). 
Hanna-Pladdy et al.: Predictors of Mild Cognitive Impairment in Early-Stage Parkinson's Disease

\section{Procedure}

All subjects were screened prior to enrollment to determine study eligibility (see table 1 for screening measures). A repeated-measures model was utilized for the study, with levodopa medication state (on vs. off) as the within-subjects factor and group (control vs. PD) as the between-subjects factor. All subjects received two alternative forms of neuropsychological assessments on two visits, with an 8-week interval between visits to minimize practice effects. The medication state was counterbalanced across visits 1 and 2 (i.e., one half of the subjects were in the on state for visit 1 , while the other half of the subjects were in the off medication state for visit 1 and vice versa).

The neuropsychological assessment included the RBANS Form A or Alternate Form B that provides subtest scores for Immediate Memory (List Learning, Story Memory), Visuospatial/Constructional (Figure Copy, Line Orientation), Language (Picture Naming, Semantic Fluency), Attention (Digit Span, Coding), and Delayed Memory (List Learning Free Recall and Recognition, and Story Memory Free Recall, Figure Free Recall) domains [25] (see table 3 for specific measures included in the comprehensive neuropsychological battery). Additional measures of attention and inhibition were provided by subtests from the Wechsler Intelligence Scale - Fourth Edition [WAIS-IV Digit Span and Letter-Number Sequencing (LNS)] and the Stroop Color and Word Test [27, 28]. Several subtests measuring visuomotor integration, motor speed and cognitive flexibility (Trails 1-5), fluency and switching, and planning functions (Tower Test) from the Delis-Kaplan Executive Function System (D-KEFS) were also administered $[27,29]$.

\section{Statistical Analyses}

Raw scores from neuropsychological tests were converted into z-scores based on the mean and SD of normal controls for each neuropsychological measure to allow for normalization across tests for comparison. Individual repeated-measures MANOVAs were conducted for the attention/executive, language, memory, and visuospatial/visuomotor function domains, with group (PD vs. controls) as the between-subjects factor and medication state (on vs. off) as the within-subjects factor. Differences in the dependent measures were initially evaluated by multivariate measures of significance and followed with univariate analyses for main effects and interactions as appropriate.

\section{Results}

\section{Subjects}

Healthy controls and PD subjects did not significantly differ in terms of age or strength of right handedness; nevertheless, controls displayed slightly higher educational levels [F(1, $80)=5.03, p<0.05$; table 1]. PD patients were nondemented based on their MMSE scores and did not display clinically significant symptoms of anxiety or depression. However, as expected, PD patients scored lower on the MMSE than controls $[\mathrm{F}(1,80)=11.69, \mathrm{p}<0.001]$ and endorsed more symptoms of anxiety $[\mathrm{F}(1,80)=42.34, \mathrm{p}<0.001]$ and depression $[\mathrm{F}(1,80)=30.97, \mathrm{p}<$ 0.001; see table 1 for means and SD]. PD patients displayed higher UPDRS total scores $(\mathrm{t}=$ $-11.12, p<0.001)$ and UPDRS motor scores $(t=-10.66, p<0.001)$ in the off state compared to the on state, verifying adequate medication washout. However, they did not differ in sleep or self-reported measures of anxiety or depression between medication states (see table 2 for means and SD). Since education differences were identified between the PD and healthy control groups, education was investigated as a covariate in all models below. 
Hanna-Pladdy et al.: Predictor Parkinson's Disease

Table 3. Neuropsychological impairment indices (z-scores) for PD subjects and significance relative to controls

\begin{tabular}{|c|c|c|c|c|}
\hline & z-scores & $\mathrm{F}$ & $\mathrm{p}$ & $\eta^{2}$ \\
\hline \multicolumn{5}{|l|}{ Attention/executive } \\
\hline WAIS-IV DST & $-0.47 \pm 1.13$ & 4.04 & 0.048 & 0.05 \\
\hline WAIS-IV DSF & $-0.21 \pm 1.04$ & 0.89 & 0.350 & 0.01 \\
\hline WAIS-IV Digit Span Backward & $-0.45 \pm 0.89$ & 4.56 & 0.036 & 0.06 \\
\hline WAIS-IV Digit Span Sequence & $-0.64 \pm 1.69$ & 4.45 & 0.038 & 0.06 \\
\hline WAIS-IV LNS & $-0.84 \pm 1.50$ & 8.91 & 0.004 & 0.11 \\
\hline D-KEFS Tower Rule Violations & $-0.86 \pm 2.15$ & 5.48 & 0.022 & 0.07 \\
\hline D-KEFS Tower Total Completion Time & $-0.84 \pm 0.98$ & 14.88 & 0.000 & 0.16 \\
\hline Stroop Word & $-0.68 \pm 1.07$ & 8.85 & 0.004 & 0.10 \\
\hline Stroop Color & $-0.25 \pm 0.96$ & 1.29 & 0.260 & 0.02 \\
\hline Stroop Color-Word & $-0.23 \pm 0.95$ & 1.16 & 0.285 & 0.02 \\
\hline \multicolumn{5}{|l|}{ Language } \\
\hline D-KEFS Letter Fluency & $-0.27 \pm 1.28$ & 1.12 & 0.294 & 0.01 \\
\hline D-KEFS Category Fluency & $-0.48 \pm 1.13$ & 4.09 & 0.047 & 0.05 \\
\hline D-KEFS Switching Fluency & $-0.54 \pm 1.27$ & 4.53 & 0.036 & 0.05 \\
\hline D-KEFS Switching Accuracy & $-0.52 \pm 1.32$ & 4.10 & 0.046 & 0.05 \\
\hline RBANS Picture Naming & $-1.45 \pm 2.41$ & 12.83 & 0.001 & 0.14 \\
\hline RBANS Semantic Fluency & $-0.55 \pm 1.13$ & 5.48 & 0.022 & 0.06 \\
\hline \multicolumn{5}{|l|}{ Memory } \\
\hline RBANS List Learning & $-1.02 \pm 1.44$ & 14.06 & 0.000 & 0.15 \\
\hline RBANS Story Immediate Memory & $-0.86 \pm 1.49$ & 9.51 & 0.003 & 0.11 \\
\hline RBANS List Recall & $-1.08 \pm 1.36$ & 17.11 & 0.000 & 0.18 \\
\hline RBANS List Recognition & $-1.41 \pm 1.82$ & 19.18 & 0.000 & 0.19 \\
\hline RBANS Story Recall & $-1.34 \pm 1.73$ & 18.78 & 0.000 & 0.19 \\
\hline RBANS Figure Recall & $-0.76 \pm 1.21$ & 9.64 & 0.003 & 0.11 \\
\hline \multicolumn{5}{|l|}{ Visuomotor/visuospatial } \\
\hline D-KEFS Trails 1 (Visual) & $-1.01 \pm 1.88$ & 9.34 & 0.003 & 0.11 \\
\hline D-KEFS Trails 2 (Number) & $-1.64 \pm 2.44$ & 16.17 & 0.000 & 0.17 \\
\hline D-KEFS Trails 3 (Letter) & $-1.80 \pm 3.36$ & 11.03 & 0.001 & 0.12 \\
\hline D-KEFS Trails 4 (Number-Letter) & $-1.79 \pm 3.36$ & 10.85 & 0.001 & 0.12 \\
\hline D-KEFS Trails 5 (Motor) & $-1.57 \pm 2.21$ & 17.38 & 0.000 & 0.18 \\
\hline RBANS Coding & $-1.04 \pm 1.48$ & 14.12 & 0.000 & 0.15 \\
\hline RBANS Line Orientation & $-0.34 \pm 0.85$ & 2.76 & 0.100 & 0.03 \\
\hline RBANS Figure Copy & $-0.70 \pm 1.24$ & 7.91 & 0.006 & 0.09 \\
\hline
\end{tabular}

$\mathrm{z}$-scores represent mean \pm SD. Significant $\mathrm{p}$ values are bolded .

\section{Attention/Executive}

The multivariate model for attention/executive subtests was significant for betweengroups differences $[\mathrm{F}(10,71)=2.97, \mathrm{p}<0.005]$; however, education did not significantly adjust the variance in dependent measures and thus was not utilized as a covariate. All attention/executive subtests were statistically significant between the groups, demonstrating lower z-scores for PD subjects compared to controls, with the exception of the WAIS-IV Digit Span Forward (DSF) and the Stroop Color and Color-Word Tests (see table 3 for univariate values). A comparison of the effect sizes revealed the greatest significance for the D-KEFS Tower Total Completion Time, followed by the WAIS-IV LNS and Stroop Word scores. However, an evaluation of the z-scores revealed a very subtle decline in the attention/executive measures for early-stage PD patients of less than 1 SD below the mean of healthy 
Hanna-Pladdy et al.: Predictors of Mild Cognitive Impairment in Early-Stage Parkinson's Disease

controls. A medication effect was only evident for the Digit Span Total [DST; F(1, 80) $=5.01$, $\left.p=0.012, \eta^{2}=0.10\right]$ and the $\operatorname{DSF}\left[F(1,80)=6.56, p=0.028, \eta^{2}=0.08\right]$. PD patients displayed significantly greater impairment on the DST $\left(\mathrm{DST}_{\text {off }}=-0.588 ; \mathrm{DST}_{\text {on }}=-0.356\right)$ and the DSF $\left(\mathrm{DSF}_{\text {off }}=-0.322 ; \mathrm{DSF}_{\text {on }}=-0.101\right)$ in the off medication state, although they did not differ from controls on the DSF and barely reached significance for the DST.

\section{Language}

The multivariate model for language subtests was significant for between-groups differences $[F(6,75)=2.94, p=0.012]$ but not for within-subjects medication effects. All language subtests were significant between-groups, with the exception of D-KEFS Letter Fluency (table 3). PD patients displayed lower scores on all language subtests; however, the RBANS Picture Naming subtest displayed the greatest level of impairment and the largest effect size, with PD patients falling -1.45 SD below the means of normal controls (table 3). Conversely, across fluency measures, PD patients were only -0.27 to -0.55 SD below normal controls. When education was utilized as a covariate, as it significantly adjusted for language functions at the multivariate level $[F(6,74)=3.26, p=0.007]$, the D-KEFS Fluency measures were no longer significant. The RBANS Picture Naming scores remained highly significant $(p<0.001)$, while the RBANS Semantic Fluency scores only approached significance $(p=0.057)$.

\section{Memory}

The multivariate model revealed a between-groups significance across memory subtests $[F(6,75)=4.52, p<0.001]$, but within-subjects effects for medication as well as education as a covariate were not significant. PD subjects scored lower than normal controls in all learning and memory measures on the RBANS, although effect sizes were the largest for Story Delayed Recall, List Recognition, and List Recall (table 3). Impairment indices based on z-scores revealed that early-stage PD patients scored from -0.76 to -1.41 SD below the mean of normal controls.

\section{Visuomotor/Visuospatial}

The multivariate model revealed between-groups significance across visuomotor/visuospatial subtests $[\mathrm{F}(8,73)=2.89, \mathrm{p}=0.007]$, but there was no within-subjects significance for medication. PD patients scored significantly below normal controls on all subtests, with the exception of RBANS Line Orientation. Effect sizes were greatest for D-KEFS Trails 5 (Motor), D-KEFS Trails 2 (Number), and RBANS Coding. However, impairment indices were greatest for D-KEFS Trails 3 and 4 (Letter and Number-Letter alternation), displaying the most significant deviation relative to controls $(-1.79$ and -1.8$)$ across the entire neuropsychological battery. Although education significantly adjusted for the variance in the model when utilized as a covariate $[F(8,72)=2.63, p=0.014]$, the significance of the subtests remained unchanged.

\section{Regression Analyses}

Based on effect sizes and impairment indices, the following neuropsychological measures from each cognitive domain were selected as stepwise predictors for a multiple regression model to identify the best predictors of cognitive impairment in early-stage PD patients relative to normal controls: LNS, D-KEFS Tower Completion Time, Stroop Word, RBANS Naming, RBANS Memory (List Learning, List Recall, Story Recall, and Figure Recall), D-KEFS Trails 2, 4, and 5, and RBANS Coding. After controlling for age and education [accounting for only $6.9 \%$ of the variance; $F(2,79)=2.91, p=0.061$ ], Trails 5 [Motor; $F(3,78)=9.31, p<$ $0.0001]$ and RBANS List Recall $[F(3,78)=10.06, p<0.0001]$ were the best predictors of earlystage PD cognitive impairment, accounting for an additional 19.5 and $7.9 \%$ of the variance, respectively. In a second regression model that eliminated visuomotor tasks (i.e., Trails), RBANS List Recall $[18.6 \%$ of the variance; $F(3,78)=8.89, \mathrm{p}<0.0001]$, LNS [an additional 
Table 4. Predictors of group (PD vs. controls) and neuropsychological performance for PD patients in the off medication state based on stepwise multiple regression analyses

\begin{tabular}{|c|c|c|c|c|c|c|}
\hline Dependent variable & & $\beta$ & $\operatorname{SE}(\beta)$ & $\beta$ & $\mathrm{t}$ & $\mathrm{p}$ \\
\hline \multirow[t]{4}{*}{ Group } & Age & -0.035 & 0.009 & -0.439 & -4.07 & 0.0001 \\
\hline & WAIS-IV LNS & -0.050 & 0.016 & -0.329 & -3.04 & 0.003 \\
\hline & RBANS List Recall & -0.073 & 0.024 & -0.329 & -2.99 & 0.004 \\
\hline & RBANS Figure Recall & -0.033 & 0.015 & -0.221 & -2.19 & 0.031 \\
\hline \multicolumn{7}{|c|}{$P D$ patients in the off medication state } \\
\hline \multirow[t]{2}{*}{ RBANS List Recall ${ }_{\text {off }}$} & Age & -0.072 & 0.017 & -0.544 & -4.21 & 0.0001 \\
\hline & PDQ-39 ${ }_{\text {off }}$ & -0.022 & 0.008 & -0.348 & -2.71 & 0.010 \\
\hline \multirow[t]{2}{*}{ RBANS Figure Recall $_{\text {off }}$} & Age & -0.087 & 0.021 & -0.587 & -4.12 & 0.0001 \\
\hline & Disease duration & 0.156 & 0.062 & 0.353 & 2.50 & 0.017 \\
\hline \multirow[t]{2}{*}{ WAIS-IV LNS off } & Age & -0.060 & 0.016 & -0.517 & -3.71 & 0.001 \\
\hline & PDQ-39 ${ }_{\text {off }}$ & -0.017 & 0.008 & -0.293 & -2.12 & 0.041 \\
\hline \multirow[t]{2}{*}{ D-KEFS Trails 5 Time $_{\text {off }}$} & Age & 0.088 & 0.023 & -0.510 & -3.84 & 0.0001 \\
\hline & $\mathrm{BAI}_{\text {off }}$ & 0.413 & 0.173 & -0.314 & -2.38 & 0.023 \\
\hline D-KEFS Tower Total Time ${ }_{\text {off }}$ & PDQ-39 $9_{\text {off }}$ & 0.032 & 0.014 & 0.342 & 2.25 & 0.031 \\
\hline
\end{tabular}

8.5\%; $F(4,77)=9.86, p<0.0001]$, and Figure Recall [an additional 3.9\%; $F(5,76)=9.24, p<$ $0.0001]$ were the best predictors and explained a combined $37.8 \%$ of the variance in terms of cognitive impairment in early-stage PD (table 4).

To expand on the above regression analyses, the performance of each significant predictor was utilized in separate regression models to determine disease-related predictors of cognitive performance in early-stage PD. The predictors entered into the equation for each model (after forcing age and education into the first step of the regression) included daily levodopa dosage, disease duration, and either on or off scores for the UPDRS Motor, Parkinson's Disease Questionnaire 39 (PDQ-39), Epworth Sleepiness Scale (EP), Parkinson's Disease Sleep Scale (PDSS), Beck Anxiety Inventory (BAI), and Beck Depression Inventory II (BDI-II). Cognitive performance was modeled both for on and off medication states, with the corresponding appropriate variables in either the on or off medication state utilized as predictors. The first set of regression analyses for the off medication state is presented in table 4 . Age and education significantly accounted for $27.1 \%$ of the variance $[F(2,37)=8.06, p<0.001]$ for RBANS List Recall in the off state, while the PDQ-39 in the off medication state explained an additional $12 \%$ of the variance $[\mathrm{F}(3,36)=8.76, \mathrm{p}<0.001]$. Following age and education [accounting for $24.4 \%$ of the variance; $F(2,37)=5.79, \mathrm{p}=0.007$ ], RBANS Figure Recall in the off state was best predicted by disease duration, explaining an additional $11.5 \%$ of the variance $[F(3,36)=6.52, p=0.001]$. WAIS-IV LNS performance in the off state was predicted by age and education [25.3\% of the variance; $F(2,37)=6.08, p=0.005]$, followed by the PDQ-39 in the off state, explaining an additional $8.5 \%$ of the variance $[F(3,36)=5.95, p=$ $0.002]$. Finally, $30.2 \%$ of the variance in the D-KEFS Trails 5 performance in the off state was explained by age and education $[\mathrm{F}(2,37)=7.79, \mathrm{p}=0.002]$, with the BAI explaining an additional $9.8 \%$ of the variance $[\mathrm{F}(3,36)=7.76, \mathrm{p}=0.001]$.

The same dependent cognitive measures were evaluated in the on medication state, but the disease predictors were not significant with the exception of D-KEFS Trails 5, which measures motor speed. After accounting for the variance from age and education [32\% of the variance; $F(2,37)=8.69, p=0.001]$, the UPDRS motor score in the on state accounted for an additional $9.6 \%$ of the variance in the D-KEFS Trails 5 performance in the on state $[\mathrm{F}(3,36)=8.56, \mathrm{p}=0.001]$. 


\section{Discussion}

Our study results reveal clear statistical differences between healthy controls and earlystage PD patients across all cognitive domains. Based on impairment indices and effect sizes, the most pronounced differences emerged for visuomotor and verbal memory functions. While impairment was also evident for working memory and planning functions, which is consistent with the expected frontostriatal cognitive dysfunction in PD, the impairment indices and effect sizes for these measures were not as impressive. Similarly, simple attentional and visuospatial functions were unimpaired, and our findings reveal that visuomotor and visuospatial functions previously attributed to cognitive decline are likely the result of motor impairment. While between-groups differences in fluency were present, these were accounted for based on educational differences. Furthermore, dopaminergic modulation as measured by differences between on and off medication states was only significant for simple attentional measures that displayed the least deviation from normal controls. Therefore, our results support the early presence of cognitive deficits across most cognitive domains; however, dopaminergic depletion was not capable of accounting for the mild cognitive deficits present at this early stage of the neurodegenerative process [7, 30, 31].

A clear segregation of cognitive deficits identified early in the course of PD, attributable to extranigral sources, and dopamine-dependentattentional and motor functions is supported by our study findings. The results indicate that cognitive deficits are present in the earliest stages of the disease prior to dopamine-mediated cognitive dysfunction and implicate an early involvement of nondopaminergic systems previously considered (e.g., noradrenergic locus coeruleus, serotonergic raphe nuclei, or early involvement of cholinergic structures in the forebrain) or alternatively structural changes in frontal and temporoparietal association cortices involved in mild cognitive deficits [32-34]. Impairment on tests of visuomotor integration, planning, and working memory functions replicate previously documented frontostriatal deficits in PD, although, apart from motor task demands, they were not the most pronounced and did not respond to dopaminergic medications. These findings are not congruent with previous descriptions of cognition in nondemented PD patients but may be partly related to differences in disease severity [35]. In summary, our findings raise the question of whether extranigral pathology is present before dopamine depletion given the presence of extensive cognitive deficits that did not differ between medication states. However, evidence of prominent memory impairment is consistent with previously identified extensive hypometabolism in temporoparietal regions in PD patients with cognitive impairment [18-20, 36].

Disease-related predictors in the regression model were not capable of explaining the cognitive impairment in the on medication state but conversely explained the cognitive variance while off medication. While these findings are suggestive of differences based on dopamine mediation, most of the variance for cognitive performance in the off medication state was explained by age and the PDQ-39, as opposed to other, more pertinent, disease predictors or levodopa dosage. A positive correlation between increasing age and cognitive dysfunction has been previously documented as being predictive of a more rapid disease progression in PD [37]. Overall, the results of the regression reflect increased health-related concerns for PD patients in the off medication state and are congruent with other reports in the literature regarding the predictive relationship between cognitive impairment in PD and quality of life [38, 39]. However, in our study, subjects were evaluated after 8 weeks between visits, and patients only discontinued medications the night before the evaluation. Thus, the relationship between the PDQ-39 and the off medication state is more likely related to a transient increase in health-related concerns rather than being indicative of the impact of cognitive deficits on sustained quality of life, as has been previously suggested $[13,38]$. Alternatively, 
Hanna-Pladdy et al.: Predictors of Mild Cognitive Impairment in Early-Stage

Parkinson's Disease

it is conceivable that transient changes in mood related to increased symptoms in the off medication state could explain our findings (this is also supported by the significant prediction of anxiety symptoms by the Trails 5 performance) as well as a relationship between PDQ-39 and mood measures which has been previously established [38]. A strong predictor of cognitive deficits in PD relative to controls was nonverbal memory recall, and this in turn was predicted by disease duration in the off medication state. These findings suggest that memory impairments in PD are progressive throughout the course of the disease, which is consistent with the amyloid burden in PD that predicts cognitive decline over time [40]. However, progressive decline in nonverbal memory following dopamine replacement therapy has been reported in de novo patients and could conceivably be an alternate explanation [41].

In summary, our investigation does not support a dopaminergic basis for early cognitive deficits in PD, and the least impaired cognitive functions were attentional tasks associated with the dorsal frontostriatal circuitry implicated in early-stage PD and dopamine depletion. Our findings are in line with investigations of mild cognitive impairment identifying greater memory than executive deficits in the early stages of PD, although there were differences in complex attentional and executive subtests with emphasis on planning and working memory functions, displaying some sensitivity to cognitive deficits related to the frontostriatal circuitry. These frontostriatal deficits have traditionally been assumed to be secondary to dopamine deficiency, but based on our results, cortical thinning identified as being particularly pronounced for frontotemporal regions in early stages of PD is a more plausible explanation $[19,20]$.

\section{Conclusions}

Overall, these findings challenge previously described stages of pathological progression and raise the question of the neuropathological basis for PD-associated cognitive deficits present in the earliest stages of the disease process that do not appear to be associated with significant dopamine depletion [7, 12, 42]. Future longitudinal studies evaluating cognitive deficits in PD with a clear identification of the relationship with both structural and functional neuroimaging findings based on disease stage or disease duration will help elucidate the extranigral basis of cognitive deficits in PD.

\section{Acknowledgements}

The project described was supported by NIH/NINDS R21NS059649 (to B.H.-P.). Data were collected in the Landon Center on Aging at the University of Kansas Medical Center, while data analyses and manuscript preparation were conducted at the Emory University Medical School, Atlanta, Ga., USA.

\section{References}

1 Hughes AJ, Ben-Shlomo Y, Daniel SE, Lees AJ: What features improve the accuracy of clinical diagnosis in Parkinson's disease: a clinicopathologic study. Neurology 1992;42:1142-1146.

$\rightarrow 2$ Parkinson J: An essay on the shaking palsy. 1817. J Neuropsychiatry Clin Neurosci 2002;14:223-236; discussion 222.

-3 Tolosa E, Gaig C, Santamaria J, Compta Y: Diagnosis and the premotor phase of Parkinson disease. Neurology 2009;72:S12-S20. 
-4 Siderowf A, Stern MB: Premotor Parkinson's disease: clinical features, detection, and prospects for treatment. Ann Neurol 2008;64(suppl 2):S139-S147.

5 Kurtz AL, Kaufer DI: Dementia in Parkinson's disease. Curr Treat Options Neurol 2011;13:242-254.

-6 Martinez-Martin P, Falup-Pecurariu C, Rodriguez-Blazquez C, Serrano-Duenas M, Carod Artal FJ, Rojo Abuin JM, Aarsland D: Dementia associated with Parkinson's disease: applying the Movement Disorder Society Task Force criteria. Parkinsonism Relat Disord 2011;17:621-624.

7 Aarsland D, Bronnick K, Williams-Gray C, Weintraub D, Marder K, Kulisevsky J, Burn D, Barone P, Pagonabarraga J, Allcock L, Santangelo G, Foltynie T, Janvin C, Larsen JP, Barker RA, Emre M: Mild cognitive impairment in Parkinson disease: a multicenter pooled analysis. Neurology 2010;75:1062-1069.

8 Park KW, Kim HS, Cheon SM, Cha JK, Kim SH, Kim JW: Dementia with Lewy bodies versus Alzheimer's disease and Parkinson's disease dementia: a comparison of cognitive profiles. J Clin Neurol 2011;7:19-24.

$\$ 9$ Cools R: Dopaminergic modulation of cognitive function - implications for L-DOPA treatment in Parkinson's disease. Neurosci Biobehav Rev 2006;30:1-23.

$\checkmark 10$ Cools R, Barker RA, Sahakian BJ, Robbins TW: Enhanced or impaired cognitive function in Parkinson's disease as a function of dopaminergic medication and task demands. Cereb Cortex 2001;11:1136-1143.

-11 Cropley VL, Fujita M, Bara-Jimenez W, Brown AK, Zhang XY, Sangare J, Herscovitch P, Pike VW, Hallett M, Nathan PJ, Innis RB: Pre- and post-synaptic dopamine imaging and its relation with frontostriatal cognitive function in Parkinson disease: PET studies with [11C]NNC 112 and [18F]FDOPA. Psychiatry Res 2008;163: 171-182.

-12 Mamikonyan E, Moberg PJ, Siderowf A, Duda JE, Have TT, Hurtig HI, Stern MB, Weintraub D: Mild cognitive impairment is common in Parkinson's disease patients with normal Mini-Mental State Examination (MMSE) scores. Parkinsonism Relat Disord 2009;15:226-231.

13 Leroi I, McDonald K, Pantula H, Harbishettar V: Cognitive impairment in Parkinson disease: impact on quality of life, disability, and caregiver burden. J Geriatr Psychiatry Neurol 2012;25:208-214.

14 Litvan I, Aarsland D, Adler CH, Goldman JG, Kulisevsky J, Mollenhauer B, Rodriguez-Oroz MC, Troster AI, Weintraub D: MDS Task Force on mild cognitive impairment in Parkinson's disease: critical review of PD-MCI. Mov Disord 2011;26:1814-1824.

15 Janvin CC, Larsen JP, Aarsland D, Hugdahl K: Subtypes of mild cognitive impairment in Parkinson's disease: progression to dementia. Mov Disord 2006;21:1343-1349. Goldman JG, Litvan I: Mild cognitive impairment in Parkinson's disease. Minerva Med 2011;102:441-459.

17 Levy G, Jacobs DM, Tang MX, Cote LJ, Louis ED, Alfaro B, Mejia H, Stern Y, Marder K: Memory and executive function impairment predict dementia in Parkinson's disease. Mov Disord 2002;17:1221-1226.

18 Stern MB, Lang A, Poewe W: Toward a redefinition of Parkinson's disease. Mov Disord 2012;27:54-60.

19 Beyer MK, Janvin CC, Larsen JP, Aarsland D: A magnetic resonance imaging study of patients with Parkinson's disease with mild cognitive impairment and dementia using voxel-based morphometry. J Neurol Neurosurg Psychiatry 2007;78:254-259.

20 Tinaz S, Courtney MG, Stern CE: Focal cortical and subcortical atrophy in early Parkinson's disease. Mov Disord 2011;26:436-441.

21 Ibarretxe-Bilbao N, Junque C, Marti MJ, Tolosa E: Brain structural MRI correlates of cognitive dysfunctions in Parkinson's disease. J Neurol Sci 2011;310:70-74.

-22 Hosokai Y, Nishio Y, Hirayama K, Takeda A, Ishioka T, Sawada Y, Suzuki K, Itoyama Y, Takahashi S, Fukuda H, Mori E: Distinct patterns of regional cerebral glucose metabolism in Parkinson's disease with and without mild cognitive impairment. Mov Disord 2009;24:854-862.

23 Oldfield RC: The assessment and analysis of handedness: The Edinburgh inventory. Neuropsychologia 1971; 9:97-113.

24 Folstein MF, Folstein SE, McHugh PR: 'Mini-mental state'. A practical method for grading the cognitive state of patients for the clinician. J Psychiatr Res 1975;12:189-198.

-25 Randolph C, Tierney MC, Mohr E, Chase TN: The Repeatable Battery for the Assessment of Neuropsychological Status (RBANS): preliminary clinical validity. J Clin Exp Neuropsychol 1998;20:310-319.

26 Hughes AJ, Daniel SE, Kilford L, Lees AJ: Accuracy of clinical diagnosis of idiopathic Parkinson's disease: a clinico-pathological study of 100 cases. J Neurol Neurosurg Psychiatry 1992;55:181-184.

27 Wechsler D (ed): Wechsler Adult Intelligence Scale, ed 4. San Antonio, Psychological Corporation, 2008.

28 Golden C: Stroop Color and Word Test. Chicago, Stoelting Company, 1978.

29 Delis DC, Kramer JH, Kaplan E, Holdnack J: Reliability and validity of the Delis-Kaplan Executive Function System: an update. J Int Neuropsychol Soc 2004;10:301-303.

30 McKinlay A, Grace RC, Dalrymple-Alford JC, Roger D: Cognitive characteristics associated with mild cognitive impairment in Parkinson's disease. Dement Geriatr Cogn Disord 2009;28:121-129.

-31 Muslimovic D, Post B, Speelman JD, Schmand B: Cognitive profile of patients with newly diagnosed Parkinson disease. Neurology 2005;65:1239-1245.

-32 Braak H, Del Tredici K, Rub U, de Vos RA, Jansen Steur EN, Braak E: Staging of brain pathology related to sporadic Parkinson's disease. Neurobiol Aging 2003;24:197-211.

-33 Pagonabarraga J, Gomez-Anson B, Rotger R, Llebaria G, Garcia-Sanchez C, Pascual-Sedano B, Gironell A, Delfino M, Ruscalleda J, Kulisevsky J: Spectroscopic changes associated with mild cognitive impairment and dementia in Parkinson's disease. Dement Geriatr Cogn Disord 2012;34:312-318. 
-34 Morales DA, Vives-Gilabert Y, Gomez-Anson B, Bengoetxea E, Larranaga P, Bielza C, Pagonabarraga J, Kulisevsky J, Corcuera-Solano I, Delfino M: Predicting dementia development in Parkinson's disease using Bayesian network classifiers. Psychiatry Res 2012, E-pub ahead of print.

-35 McKinlay A, Grace RC, Dalrymple-Alford JC, Roger D: Characteristics of executive function impairment in Parkinson's disease patients without dementia. J Int Neuropsychol Soc 2010;16:268-277.

-36 Huang C, Ravdin LD, Nirenberg MJ, Piboolnurak P, Severt L, Maniscalco JS, Solnes L, Dorfman BJ, Henchcliffe C: Neuroimaging markers of motor and nonmotor features of Parkinson's disease: an $\left[{ }^{18} \mathrm{~F}\right]$ fluorodeoxyglucose positron emission computed tomography study. Dement Geriatr Cogn Disord 2013;35:183-196.

-37 Velseboer DC, Broeders M, Post B, van Geloven N, Speelman JD, Schmand B, de Haan RJ, de Bie RM: Prognostic factors of motor impairment, disability, and quality of life in newly diagnosed PD. Neurology 2013;80:627633.

-38 Schrag A, Jahanshahi M, Quinn N: What contributes to quality of life in patients with Parkinson's disease? J Neurol Neurosurg Psychiatry 2000;69:308-312.

39 Witt K, Daniels C, Krack P, Volkmann J, Pinsker MO, Kloss M, Tronnier V, Schnitzler A, Wojtecki L, Botzel K, Danek A, Hilker R, Sturm V, Kupsch A, Karner E, Deuschl G: Negative impact of borderline global cognitive scores on quality of life after subthalamic nucleus stimulation in Parkinson's disease. J Neurol Sci 2011;310: 261-266.

40 Gomperts SN, Locascio JJ, Rentz D, Santarlasci A, Marquie M, Johnson KA, Growdon JH: Amyloid is linked to cognitive decline in patients with Parkinson disease without dementia. Neurology 2013;80:85-91.

41 Miah IP, Olde Dubbelink KT, Stoffers D, Deijen JB, Berendse HW: Early-stage cognitive impairment in Parkinson's disease and the influence of dopamine replacement therapy. Eur J Neurol 2012;19:510-516.

-42 Pappata S, Santangelo G, Aarsland D, Vicidomini C, Longo K, Bronnick K, Amboni M, Erro R, Vitale C, Caprio MG, Pellecchia MT, Brunetti A, De Michele G, Salvatore M, Barone P: Mild cognitive impairment in drug-naive patients with PD is associated with cerebral hypometabolism. Neurology 2011;77:1357-1362. 